\title{
Multiple solutions of indefinite elliptic systems via a Galerkin-type Conley index theory
}

\author{
by \\ Marek Izydorek (Gdańsk) and \\ Krzysztof P. Rybakowski (Rostock)
}

\begin{abstract}
Let $\Omega$ be a bounded domain in $\mathbb{R}^{N}$ with smooth boundary. Consider the following elliptic system:

$$
\begin{aligned}
-\Delta u & =\partial_{v} H(u, v, x) & & \text { in } \Omega, \\
-\Delta v & =\partial_{u} H(u, v, x) & & \text { in } \Omega, \\
u & =0, \quad v=0 & & \text { in } \partial \Omega .
\end{aligned}
$$

We assume that $H$ is an even "-"-type Hamiltonian function whose first order partial derivatives satisfy appropriate growth conditions. We show that if $(0,0)$ is a hyperbolic solution of (ES), then (ES) has at least $2|\mu|$ nontrivial solutions, where $\mu=\mu(0,0)$ is the renormalized Morse index of $(0,0)$. This proves a conjecture by Angenent and van der Vorst.
\end{abstract}

1. Introduction. Let $\Omega$ be a bounded domain in $\mathbb{R}^{N}$ with smooth boundary. Consider the following elliptic system

$$
\begin{aligned}
-\Delta u=\partial_{v} H(u, v, x) & & \text { in } \Omega, \\
-\Delta v=\partial_{u} H(u, v, x) & & \text { in } \Omega, \\
u=0, \quad v=0 & & \text { in } \partial \Omega .
\end{aligned}
$$

Throughout this section we make the following

Hypothesis 1.1. (1) $p, q \in] 1, \infty[$ are such that $1 / p>1 / 2-2 / N, 1 / q>$ $1 / 2-2 / N$ and $1 / p+1 / q>1-2 / N$.

(2) The function $H: \mathbb{R} \times \mathbb{R} \times \bar{\Omega} \rightarrow \mathbb{R},(\xi, \eta, x) \mapsto H(\xi, \eta, x)$, is of class $C^{2}$. $58 \mathrm{E} 50$.

2000 Mathematics Subject Classification: Primary 35J50, 58E40; Secondary 58E05,

Key words and phrases: equivariant Conley index, renormalized Morse index, Angenent-van der Vorst conjecture. 
(3) There is a constant $\left.c_{1} \in\right] 0, \infty[$ such that for all $(\xi, \eta, x) \in \mathbb{R} \times \mathbb{R} \times \bar{\Omega}$,

$$
\begin{aligned}
& \left|\partial_{\xi} H(\xi, \eta, x)\right| \leq c_{1}\left(|\xi|^{p-1}+|\eta|^{(p-1) q / p}+1\right), \\
& \left|\partial_{\eta} H(\xi, \eta, x)\right| \leq c_{1}\left(|\eta|^{q-1}+|\xi|^{(q-1) p / q}+1\right) .
\end{aligned}
$$
$\times \bar{\Omega}$

(4) There are constants $\left.c_{2}, \delta \in\right] 0, \infty[$ such that for all $(\xi, \eta, x) \in \mathbb{R} \times \mathbb{R}$

$$
\partial_{\xi} H(\xi, \eta, x) \xi-\partial_{\eta} H(\xi, \eta, x) \eta \geq-c_{2}+\delta\left(|\xi|^{p}+|\eta|^{q}\right) .
$$

Under the above hypothesis, system (1.1) was investigated in the recent important paper [1] by Angenent and van der Vorst. These authors show that there is a Hilbert space $E$ of functions $z=(u, v)$, a linear $E$-symmetric isometry $L: E \rightarrow E$ and a nonlinear continuous operator $K_{1}: E \rightarrow E$ such that the classical solutions of (1.1) are exactly the equilibria of the ordinary differential equation

$$
\dot{z}=L z+K_{1}(z)
$$

on $E$. Moreover, (1.2) is gradient-like with respect to a strongly indefinite functional $\Phi_{1}: E \rightarrow \mathbb{R}$. (Here we are not using the notation of [1] but, rather, the notation of our previous paper [13].) Using a specially developed version of Morse-Floer homology, Angenent and van der Vorst established a few results, which can be summarized in the following

ThEOREM A (see [1]). Suppose $z_{0}=(0,0)$ is a hyperbolic equilibrium of (1.2) with renormalized Morse index $\mu\left(z_{0}\right) \neq 0$. Then

(1) (1.1) has a nontrivial solution.

(2) If $\Phi_{1}$ is a Morse function then (1.1) has at least two nontrivial solutions.

(3) If $\Phi_{1}$ is a Morse function and $H$ is an even function, then (1.1) has at least $2\left|\mu\left(z_{0}\right)\right|$ nontrivial solutions.

The authors also conjecture that the last part of Theorem $\mathrm{A}$ is true without the assumption that $\Phi_{1}$ be Morse:

Conjecture A (see [1]). Suppose that $z_{0}=(0,0)$ is a hyperbolic equilibrium of (1.2). If $H$ is an even function, then (1.1) has at least $2\left|\mu\left(z_{0}\right)\right|$ nontrivial solutions.

In a previous paper [13] we studied the above system using a Galerkin type version of Conley index theory for equations like (1.2), developed in the article [12] (and called the $\mathcal{L S}$-Conley index there). Let us briefly recall the definition of the $\mathcal{L} \mathcal{S}$-Conley index for the particular case of equations like (1.2). To this end, recall that in [13] we defined a sequence $P^{l}: E \rightarrow E$, $l \in \mathbb{N}$, of $E$-orthogonal projectors such that, for every $l \in \mathbb{N}$, the subspace $P^{l}(E)$ is finite-dimensional and $L$-invariant, and $P^{l} z \rightarrow z$ in $E$ for every $z \in E$. Now suppose that $K: E \rightarrow E$ is a continuous map and $N$ is a closed 
bounded subset of $E$ such that the closure of $K(N)$ in $E$ is compact. For every $l \in \mathbb{N}$ define the map $g_{l}: P^{l}(E) \rightarrow P^{l}(E)$ by

$$
g_{l}(z):=L z+P^{l} K(z), \quad z \in P^{l}(E) .
$$

If $N$ is a isolating neighborhood with respect to the ordinary differential equation

$$
\dot{z}=L z+K(z)
$$

on $E$, then there is a smallest $l_{0} \in \mathbb{N}$ such that, for every $l \geq l_{0}$, the set $N \cap P^{l}(E)$ is an isolating neighborhood relative to the ordinary differential equation

$$
\dot{z}=g_{l}(z)
$$

on $P^{l}(E)$. Thus, for every $l \geq l_{0}$, the (finite-dimensional) Conley index $h\left(g_{l}, N \cap P^{l}(E)\right)$ is defined. Now we define the $\mathcal{L} \mathcal{S}$-Conley index $h(L+K, N)$ as the sequence

$$
h(L+K, N):=\left(h(L+K, N)_{l}\right)_{l \geq l_{0}}
$$

where

$$
h(L+K, N)_{l}:=h\left(g_{l}, N \cap P^{l}(E)\right), \quad l \geq l_{0} .
$$

In other words, the $\mathcal{L S}$-Conley index of an isolating neighborhood $N$ relative to the infinite-dimensional ODE (1.3) is the sequence of the Conley indices of $N$ with respect to the finite-dimensional Galerkin approximations (1.4) of (1.3). This index enjoys the usual properties of the classical Conley index, like the nontriviality and homotopy invariance property. Using this index, we established the results summarized in the following

Theorem B (see [13]). Let $z_{0}=\left(u_{0}, v_{0}\right)$ be a hyperbolic equilibrium of (1.2). Then there is a number $\gamma=\gamma\left(z_{0}\right) \in \mathbb{Z}$ such that the $\mathcal{L S}$-Conley index $h\left(L+K_{1},\left\{z_{0}\right\}\right)$ has the property that

$$
h\left(L+K_{1},\left\{z_{0}\right\}\right)_{l}=\Sigma^{\gamma+l} \quad \text { for all } l \in \mathbb{N} \text { large enough } .
$$

If , in addition, $z_{0}=(0,0)$ and $\gamma \neq 0$, then (1.1) has at least one nontrivial solution.

Here, as usual, we use the symbol $\Sigma^{r}$ to denote the homotopy type of a pointed $r$-dimensional sphere $\left(S^{r}, s_{0}\right)$.

In [4] M. Carbinatto and the second author developed a theory of Morse decompositions for equations like (1.2). As an application of a corresponding Morse equation, they established the following result:

Theorem C (see [4]). Suppose $z_{0}=(0,0)$ is a hyperbolic equilibrium of (1.2) with $\gamma=\gamma\left(z_{0}\right) \neq 0$. Moreover, suppose that $\Phi_{1}$ is a Morse function. Then (1.1) has at least two nontrivial solutions. If, in addition, $H$ is an even function, then (1.1) has at least $2|\gamma|$ nontrivial solutions. 
In this paper we complete the picture composed of the above results.

In fact, we first show, in Theorem 2.3 below, that the number $\gamma\left(z_{0}\right)$ of Theorem B equals the renormalized Morse index $\mu\left(z_{0}\right)$. This gives, firstly, a new interpretation of the renormalized Morse index. Secondly, this result implies that Theorems B and $\mathrm{C}$ together are equivalent to Theorem A. As a consequence we see that Theorem A can be proved by using Conley index methods rather than the (analytically more involved) Floer homology.

The second main result of this paper is a proof of Conjecture A (see Theorem 4.5 below). We obtain the proof by combining the $G$-equivariant version of the Galerkin type Conley index from [12] with the Morse decomposition theory from [4] and some abstract ideas from the paper [11] by the first author and the paper [8] by Floer and Zehnder.

Some of our results are of an abstract character and, properly extended and modified, they can be applied to establish multiplicity results for various other classes of strongly indefinite equivariant problems. This will be treated in a subsequent publication. For some other applications of the Conley index theory to strongly indefinite problems see [9] and [10]. Various applications of classical equivariant Conley index to problems with symmetries are contained in the lecture notes [2].

In this paper we denote by $\mathbb{R}, \mathbb{Z}, \mathbb{N}$ and $\mathbb{N}_{0}$ the sets of all real, integer, positive integer and nonnegative integer numbers, respectively. We also write $\llbracket a, b \rrbracket:=[a, b] \cap \mathbb{Z}$ for $a, b \in \mathbb{R}$. Given a topological space $X$ and $Y \subset X$, we write $\operatorname{Int}_{X}(Y)$ and $\mathrm{Cl}_{X}(Y)$ to denote the interior and closure of $Y$ in $X$, respectively. Given topological spaces $X_{1}$ and $X_{2}$ we denote by $C\left(X_{1} \rightarrow X_{2}\right)$ the set of all continuous maps from $X_{1}$ to $X_{2}$.

Finally, note that all vector spaces considered in this paper are over the reals.

2. A proof that $\gamma\left(z_{0}\right)=\mu\left(z_{0}\right)$. In this section we use the notation and results of our previous paper [13]. In particular, let the operators $A^{\alpha}, \alpha \in \mathbb{R}$, the Hilbert space $E$, the operator $L: E \rightarrow E$, the projectors $P^{l}: E \rightarrow E$, $l \in \mathbb{N}$, and the nonlinear map $K_{1}: E \rightarrow E$ be defined as in that paper. Finally, as in [13] we use the notation $h(f, S)=\left(h(f, S)_{l}\right)_{l \geq l_{0}}$ for the $\mathcal{L} \mathcal{S}$ Conley index of the pair $(f, S)$ (defined in [12]).

Recall the following

Definition 2.1. Suppose $z_{0}=\left(u_{0}, v_{0}\right) \in E$ is a solution of (1.1), i.e. $L z_{0}+K_{1}\left(z_{0}\right)=0$. Define the linear map $K_{\operatorname{lin}, z_{0}}: E \rightarrow E$ by

$$
K_{\operatorname{lin}, z_{0}}(u, v)=\left(A^{-2 s}(-a(\cdot) u+c(\cdot) v), A^{-2 t}(c(\cdot) u-b(\cdot) v)\right) .
$$

Here, the functions $a, b, c \in C(\bar{\Omega} \rightarrow \mathbb{R})$ are defined, for $x \in \bar{\Omega}$, by

$$
a(x)=\partial_{\xi \xi} H\left(z_{0}(x), x\right),
$$




$$
\begin{aligned}
b(x) & =\partial_{\eta \eta} H\left(z_{0}(x), x\right), \\
-c(x) & =\partial_{\xi \eta} H\left(z_{0}(x), x\right)=\partial_{\eta \xi} H\left(z_{0}(x), x\right) .
\end{aligned}
$$

We call $z_{0}$ hyperbolic if the linear operator $L+K_{\operatorname{lin}, z_{0}}$ is injective.

Remark. Note that the operator $L+K_{\operatorname{lin}, z_{0}}$ is the "formal" Fréchet derivative of $L+K_{1}$ at $z_{0}$. In general, the true Fréchet derivative of $L+K_{1}$ at $z_{0}$ does not exist.

The following result has been established in [13]:

Theorem 2.2 (cf. the proof of Theorem 2.9, Corollary 2.10, and Theorem 2.6 of [13]). Let $z_{0}$ and $K_{\text {lin }}=K_{\text {lin, } z_{0}}$ be as in Definition 2.1. Then $\left\{z_{0}\right\}$ is an isolated invariant set relative to $L+K_{1},\{0\}$ is an isolated invariant set relative to $L+K_{\operatorname{lin}}$ and there is an integer $\gamma=\gamma\left(z_{0}\right)$ such that $h\left(L+K_{1},\left\{z_{0}\right\}\right)_{l}=h\left(L+K_{\text {lin }},\{0\}\right)_{l}=\Sigma^{\gamma+l}$ for all $l \in \mathbb{N}$ large enough. Moreover, $\{0\}$ is an isolated invariant set relative to $L$ and $h(L,\{0\})_{l}=\Sigma^{l}$ for all $l \in \mathbb{N}$.

The renormalized Morse index of a hyperbolic solution $z_{0}$ of (1.1) was defined of [1]. To recall the definition, let us first define, for every $k \in \mathbb{N}_{0}$ and $\beta \in] 0,1\left[\right.$, the space $h^{k, \beta}(\bar{\Omega})$ to be the closure in $C^{k, \beta}(\bar{\Omega})$ of the subset of smooth functions. Given $\alpha \in] 0,1[$ set

$$
X_{\alpha}=h_{0}^{2 \alpha}(\bar{\Omega}) \times h_{0}^{2(1-\alpha)}(\bar{\Omega}) .
$$

Here,

(1) if $\beta \in] 0,1 / 2\left[\right.$, then $h_{0}^{2 \beta}(\bar{\Omega}):=\left\{\varphi \in h^{0,2 \beta}(\bar{\Omega})|\varphi|_{\partial \Omega}=0\right\}$;

(2) if $\beta=1 / 2$, then $h_{0}^{2 \beta}(\bar{\Omega}):=\left\{\varphi \in C^{1}(\bar{\Omega})|\varphi|_{\partial \Omega}=0\right\}$;

(3) if $\beta \in] 1 / 2,1\left[\right.$, then $h_{0}^{2 \beta}(\bar{\Omega}):=\left\{\varphi \in h^{1,2 \beta-1}(\bar{\Omega})|\varphi|_{\partial \Omega}=0\right\}$.

Let $\alpha=s / 2$. Note that $X_{\alpha} \subset E$ with continuous inclusion. It turns out that $L\left(X_{\alpha}\right) \subset X_{\alpha}$. Let $\widetilde{L}: X_{\alpha} \rightarrow X_{\alpha}$ be the restriction of $L$ to $X_{\alpha}$.

Now let $B_{-}$and $B_{+}$be linear, compact and $E$-symmetric operators on $X_{\alpha}$ such that $-\widetilde{L}+B_{-}$and $-\widetilde{L}+B_{+}$are injective. Let $B: \mathbb{R} \rightarrow \mathcal{L}\left(X_{\alpha}, X_{\alpha}\right)$ be an arbitrary continuous family of compact $E$-symmetric operators with

$$
\lim _{t \rightarrow-\infty}\left\|B(t)-B_{-}\right\|_{\mathcal{L}\left(X_{\alpha}, X_{\alpha}\right)}=0 \quad \text { and } \quad \lim _{t \rightarrow \infty}\left\|B(t)-B_{+}\right\|_{\mathcal{L}\left(X_{\alpha}, X_{\alpha}\right)}=0 .
$$

Then the map

$$
\Phi=\frac{d}{d t}-\widetilde{L}+B(t): W^{1,2}\left(\mathbb{R}, X_{\alpha}\right) \rightarrow L^{2}\left(\mathbb{R}, X_{\alpha}\right)
$$

is a well defined Fredholm operator. Its Fredholm index Ind $\Phi$ depends only on the pair $\left(-\widetilde{L}+B_{-},-\widetilde{L}+B_{+}\right)$and we write $\mu\left(-\widetilde{L}+B_{-},-\widetilde{L}+B_{+}\right):=\operatorname{Ind} \Phi$. Now let $z_{0}$ and $K_{\operatorname{lin}, z_{0}}: E \rightarrow E$ be as in Definition 2.1. One shows that $K_{\operatorname{lin}, z_{0}}\left(X_{\alpha}\right) \subset X_{\alpha}$. Let $\widetilde{K}: X_{\alpha} \rightarrow X_{\alpha}$ be the restriction of $K_{\operatorname{lin}, z_{0}}$ to $X_{\alpha}$. It 
is clear that both $K_{\operatorname{lin}, z_{0}}$ and $\widetilde{K}$ are compact and $E$-symmetric. Moreover, the operators $-L,-\widetilde{L},-L-K_{\operatorname{lin}, z_{0}}$ and $-\widetilde{L}-\widetilde{K}$ are injective. The renormalized Morse index $\mu\left(z_{0}\right)$ is now defined by $\mu\left(z_{0}\right)=\mu(-\widetilde{L}-\widetilde{K},-\widetilde{L}$ ) (see Definition 17 of [1]).

We can now state our first basic result.

Theorem 2.3. Assume the hypotheses of Theorem 2.2. Then $\gamma\left(z_{0}\right)=$ $\mu\left(z_{0}\right)$.

Proof. Note that for every $l \in \mathbb{N}$ the projection operator $P^{l}$ is compact, $E$-symmetric, $P^{l}\left(X_{\alpha}\right) \subset X_{\alpha}$ and the restriction of $P^{l}$ to $X_{\alpha}$ is compact. It follows that the operator $P^{l} \circ K_{\operatorname{lin}, z_{0}} \circ P^{l}: E \rightarrow E$ is $E$-symmetric and compact. It restricts to a compact operator $\widetilde{K}^{l}: X_{\alpha} \rightarrow X_{\alpha}$. Moreover, a simple compactness argument proves that there is an $l_{1} \in \mathbb{N}$ such that for all $l \geq l_{1}$ and $t \in[0,1]$ the operator $L+t K_{\operatorname{lin}, z_{0}}+(1-t) P^{l} \circ K_{\operatorname{lin}, z_{0}} \circ P^{l}: E \rightarrow E$ is injective. By Lemma 18 of [1] we thus obtain, for $l \geq l_{1}$,

$$
\mu\left(-\widetilde{L}-\widetilde{K},-\widetilde{L}-\widetilde{K}^{l}\right)=0 .
$$

The cocycle property of the relative index $\mu$ (Lemma 16 of [1]) implies that

$$
\mu(-\widetilde{L}-\widetilde{K},-\widetilde{L})=\mu\left(-\widetilde{L}-\widetilde{K}^{l},-\widetilde{L}\right), \quad l \geq l_{1} .
$$

Fix $l \geq l_{1}$ arbitrarily. Note that $E^{l}=P^{l}(E)$ is a closed subspace of $X_{\alpha}$. Let $Y^{l}$ be the intersection of $X_{\alpha}$ with the orthogonal complement of $E^{l}$ in $E$. Then $X_{\alpha}$ is the direct sum of $E^{l}$ and $Y^{l}$. Moreover, the spaces $E^{l}$ and $Y^{l}$ are invariant relative to $\widetilde{L}$ and $\widetilde{K}^{l}$ with $\left.\widetilde{L}\right|_{E^{l}}=L^{l},\left.\widetilde{K}^{l}\right|_{E^{l}}=\left.P^{l} \circ K\right|_{E^{l}}$ and $\left.\widetilde{K}^{l}\right|_{Y^{l}}=0$. Define $B^{l}(t): E^{l} \rightarrow E^{l}$ by $B^{l}(t)=-\left.\widetilde{K}^{l}\right|_{E^{l}}$ for $\left.\left.t \in\right]-\infty, 0\right]$, $B^{l}(t)=-\left.(1-t) \widetilde{K}^{l}\right|_{E^{l}}$ for $t \in[0,1]$ and $B^{l}(t)=0$ for $t \in[0, \infty[$.

Let

$$
\begin{aligned}
& \Phi_{E^{l}}=\frac{d}{d t}-\left.\widetilde{L}\right|_{E^{l}}+B^{l}(t): W^{1,2}\left(\mathbb{R}, E^{l}\right) \rightarrow L^{2}\left(\mathbb{R}, E^{l}\right), \\
& \Phi_{Y^{l}}=\frac{d}{d t}-\left.\widetilde{L}\right|_{Y^{l}}: W^{1,2}\left(\mathbb{R}, Y^{l}\right) \rightarrow L^{2}\left(\mathbb{R}, Y^{l}\right)
\end{aligned}
$$

and $\Phi=\Phi_{E^{l}} \oplus \Phi_{Y^{l}}$. Proceeding almost verbatim as in the proof of Lemma 15 of [1] we see that $\Phi_{Y^{l}}$ is invertible so $\Phi_{Y^{l}}$ is Fredholm and

$$
\text { Ind } \Phi_{Y^{l}}=0 \text {. }
$$

Now, since $E^{l}$ is finite-dimensional, it follows that $\Phi_{E^{l}}$ is Fredholm and

$$
\begin{aligned}
\operatorname{Ind} \Phi_{E^{l}} & =m_{-}\left(-L^{l}-\left.P^{l} \circ K\right|_{E^{l}}\right)-m_{-}\left(-L^{l}\right) \\
& =m_{+}\left(L^{l}+\left.P^{l} \circ K\right|_{E^{l}}\right)-m_{+}\left(L^{l}\right),
\end{aligned}
$$

where for a linear map $A$ on $E^{l}, m_{-}(A)$ (resp. $m_{+}(A)$ ) denotes the number of negative (resp. positive) eigenvalues of $A$, counted with multiplicities (cf. [14] 
or [17]). Thus $\Phi$ is Fredholm and

$$
\mu\left(-\widetilde{L}-\widetilde{K}^{l},-\widetilde{L}\right)=\operatorname{Ind} \Phi=\operatorname{Ind} \Phi_{E^{l}}+\operatorname{Ind} \Phi_{Y^{l}}=\operatorname{Ind} \Phi_{E^{l}} .
$$

Now, the definition of the $\mathcal{L S}$-Conley index from [12] and Theorem 2.2 imply that $m_{+}\left(L^{l}+\left.P^{l} \circ K\right|_{E^{l}}\right)=\gamma+l$ and $m_{+}\left(L^{l}\right)=l$ for all $l$ large enough. The theorem now follows from formulas (2.1)-(2.4).

It should be noted that the results stated so far in this section are valid under the sole Hypothesis 1.1(1)-(3).

REMARK. We now see that, under the full Hypothesis 1.1 and with the notation of the introduction, the conjunction of Theorems $\mathrm{B}$ and $\mathrm{C}$ is, indeed, equivalent to Theorem A.

In what follows we will require

Definition 2.4 (see [4]). Let $X$ be a Banach space, $U \subset X$ be open, and $f \in C(U \rightarrow X)$ be arbitrary. Let $S \subset U$ be invariant relative to $f$. A finite sequence $\left(M_{r}\right)_{r=1}^{k}$ of subsets of $S$ is called a Morse decomposition of $S$ relative to $f$ if the following properties hold:

(1) The sets $M_{r}, r \in \llbracket 1, k \rrbracket$, are closed, invariant relative to $f$ and pairwise disjoint.

(2) For every solution $\sigma$ of $f$ with $\sigma(\mathbb{R}) \subset S$, either $\sigma(\mathbb{R}) \subset M_{i}$ for some $i \in \llbracket 1, k \rrbracket$ or else there are $i, j \in \llbracket 1, k \rrbracket$ with $i<j, \alpha(\sigma) \subset M_{j}$ and $\omega(\sigma) \subset M_{i}$. Here,

$$
\left.\left.\alpha(\sigma)=\bigcap_{t \in]-\infty, 0]} \mathrm{Cl}_{X} \sigma(]-\infty, t\right]\right), \quad \omega(\sigma)=\bigcap_{t \in[0, \infty[} \mathrm{Cl}_{X} \sigma([t, \infty[)
$$

are the $\alpha$ - and $\omega$-limit sets of $\sigma$, respectively.

\section{Equivariant cohomology and an abstract multiplicity result.} In this section, in a step toward the proof of Conjecture A, we establish an abstract multiplicity result for finite-dimensional ordinary differential equations with a $\mathbb{Z}_{2}$-symmetry (see Theorem 3.8 below). This result requires some background material on transformation groups, which we briefly collect here for the readers' convenience. See [3] and [6] for general information on the subject.

Let $G$ be a compact Lie group, $E G$ be a contractible space with a free (left) $G$-action and let $B G=E G / G$ be the corresponding orbit space. Let $R$ be a commutative ring with unity and let $\left(H^{q}\right)_{q \in \mathbb{Z}}$ be the Alexander-Spanier cohomology functor with coefficients in $R$. (See [18].) As usual, if $\left(Y, Y^{\prime}\right)$ is a topological pair, then we write $H^{*}\left(Y, Y^{\prime}\right):=\bigoplus_{q \in \mathbb{Z}} H^{q}\left(Y, Y^{\prime}\right)$. We also write $H^{*}(Y):=H^{*}(Y, \emptyset)$. It is clear that $H^{*}(B G)$ is a (graded) ring with unity, where the multiplication in $H^{*}(B G)$ is defined by the cup product $\cup$. In particular, if $a \in H^{n}(B G)$ and $b \in H^{m}(B G)$, then $a b:=a \cup b \in H^{n+m}(B G)$. 
Given a (left) $G$-space $Y$ let the product $E G \times Y$ carry the diagonal action and let $Y_{G}=(E G \times Y) / G$ be the corresponding orbit space.

Let $(X, A)$ be an arbitrary pair of $G$-spaces, i.e. $X$ is a $G$-space and $A$ is a $G$-invariant subspace of $X$. Since the quotient map $E G \times X \rightarrow(E G \times X) / G$ is open, it follows that the topology of $(E G \times A) / G$ is just the subspace topology of $(E G \times A) / G$ in $(E G \times X) / G$. The $G$-equivariant cohomology of $(X, A)$ is defined as $H_{G}^{*}(X, A):=H^{*}\left(X_{G}, A_{G}\right)$. In particular, $H_{G}^{*}(X)=$ $H_{G}^{*}(X, \emptyset)$. (Notice at this point that this definition of $H_{G}^{*}(X)$, identical to that of [6], differs from the definition of $H_{G}^{*}(X)$ as made in [11].) There is a multiplication $*: H^{*}(B G) \times H_{G}^{*}(X, A) \rightarrow H_{G}^{*}(X, A)$ such that whenever $n, m \in \mathbb{N}_{0}$, then $a * u=\gamma_{X}^{*}(a) \cup u \in H_{G}^{n+m}(X, A)$ for $a \in H^{n}(B G)$ and $u \in H_{G}^{m}(X, A)$. Here, $\gamma_{X}:(E G \times X) / G \rightarrow B G$ is induced by the projection map $E G \times X \rightarrow E G$, and $\cup$ is again the cup product. This makes $H_{G}^{*}(X, A)$ into a (graded) module over the ring $H^{*}(B G)$.

Proposition 3.1. Assume that $(X, A, B)$ is a triple of $G$-spaces, i.e. $X$ is a $G$-space, $X \supset A \supset B$ and $A$ and $B$ are $G$-invariant. Let

$$
\ldots \stackrel{\delta^{*}}{\rightarrow} H_{G}^{*}(X, A) \stackrel{j^{*}}{\rightarrow} H_{G}^{*}(X, B) \stackrel{i^{*}}{\rightarrow} H_{G}^{*}(A, B) \stackrel{\delta^{*}}{\rightarrow} H_{G}^{*}(X, A) \stackrel{j^{*}}{\rightarrow} \ldots
$$

be the long exact (ordinary) cohomology sequence of the triple $\left(X_{G}, A_{G}, B_{G}\right)$. Then $j^{*}$ and $i^{*}$ are $H^{*}(B G)$-homomorphisms while $\delta^{*}(\omega * u)=(-1)^{p} \omega *$ $\delta^{*}(u)$ whenever $\omega \in H^{p}(B G)$ and $u \in H_{G}^{*}(A, B)$.

Proof. If $\left(Y, Y^{\prime}\right)$ and $\left(Z, Z^{\prime}\right)$ are topological pairs and $f:\left(Y, Y^{\prime}\right) \rightarrow\left(Z, Z^{\prime}\right)$ is continuous then $f^{*}(a \cup b)=f^{*}(a) \cup f^{*}(b)$ for all $a \in H^{*}(Z)$ and $b \in$ $H^{*}\left(Z, Z^{\prime}\right)$. This immediately implies that $j^{*}$ and $i^{*}$ are $H^{*}(B G)$-homomorphisms. Furthermore, in the notation of Section 6.5 of [18], if $\phi_{1} \in$ $\bar{C}^{p}(Y, \mathbb{R})$ and $\phi_{2} \in \bar{C}^{q}(Y, \mathbb{R})$ then $\delta\left(\phi_{1} \cup \phi_{2}\right)=\delta \phi_{1} \cup \phi_{2}+(-1)^{p} \phi_{1} \cup \delta \phi_{2}$. This easily implies the last statement of the proposition (cf. the proof of Theorem 5.3.5 of [18]).

Proposition 3.2 (cf. the proof of Remark 3.8 of [11]). Let $X$ be a $G$ space and $\omega \in H^{*}(B G)$. Then $\omega * H_{G}^{*}(X)=0$ if and only if $\gamma_{X}^{*}(\omega)=0$ in $H_{G}^{*}(X)$. Moreover, if $\omega * H_{G}^{*}(X)=0$ and $A \subset Y \subset X$ are $G$-invariant, then $\omega * H_{G}^{*}(Y, A)=0$.

Proof. Suppose $\omega * H_{G}^{*}(X)=0$ and let $e \in H^{*}(B G)$ be the unity of $H^{*}(B G)$. Then $0=\omega * \gamma_{X}^{*}(e)=\gamma_{X}^{*}(\omega) \cup \gamma_{X}^{*}(e)=\gamma_{X}^{*}(\omega \cup e)=\gamma_{X}^{*}(\omega)$, so $\gamma_{X}^{*}(\omega)=0$ in $H_{G}^{*}(X)$. Conversely, if $\gamma_{X}^{*}(\omega)=0$ in $H_{G}^{*}(X)$ then, for every $u \in H_{G}^{*}(X)$,

$$
\omega * u=\gamma_{X}^{*}(\omega) \cup u=0 \cup u=0,
$$

so $\omega * H_{G}^{*}(X)=0$. If $A \subset Y \subset X$ are $G$-invariant and $\nu: E G \times Y \rightarrow E G \times X$ 
is the inclusion induced map, then $\gamma_{X} \circ \nu=\gamma_{Y}$, so

$$
\gamma_{Y}^{*}=\nu^{*} \circ \gamma_{X}^{*} \text {. }
$$

Now, if $\omega * H_{G}^{*}(X)=0$, then by the first part of this proposition, we have $\gamma_{X}^{*}(\omega)=0$ and so formula (3.2) implies that $\gamma_{Y}^{*}(\omega)=0$ in $H_{G}^{*}(Y)$. As in the proof of formula (3.1) we now see that $\omega * u=0$ for every $u \in H_{G}^{*}(Y, A)$, so $\omega * H_{G}^{*}(Y, A)=0$.

For the rest of this paper we make the following

Standing Assumption. $G$ is the additive group $\mathbb{Z}_{2}, R$ is the ring $\mathbb{Z}_{2}$, $E G$ is the infinite-dimensional sphere $S^{\infty}$ with the antipodal action $(g, x) \mapsto$ $(-1)^{g} x$ and so $B G=\mathbb{R} P^{\infty}$, the infinite-dimensional real projective space.

It is well known (cf. Theorem III 2.5 of $[6]$ ) that $H^{*}(B G)=\mathbb{Z}_{2}[\omega]$, where $\omega$ is the (uniquely determined) generator of $H^{1}(B G)$.

Proposition 3.3. Let $X$ be a normed vector space which is also a $G$ space and $x$ be a point of $X$ with trivial isotropy group $G_{x}$. Then every neighborhood of the $G$-orbit $G x$ contains a closed $G$-invariant neighborhood $N$ of $G x$ with $H_{G}^{q}(N)=0$ for all $q \in \mathbb{N}$. In particular, $\omega * H_{G}^{*}(N)=0$.

Proof. Since the isotropy group $G_{x}$ is trivial, it follows that $G x$ contains exactly two points, namely $x$ and $\Theta(x)$, where $\Theta: X \rightarrow X$ is the left translation corresponding to the element $1 \in \mathbb{Z}_{2}$. Thus there is a $G$ equivariant homeomorphism $\alpha: G x \rightarrow G, g x \mapsto g$. The map $\operatorname{Id}_{E G} \times \alpha$ thus induces a homeomorphism $(E G \times G x) / G \rightarrow(E G \times G) / G$. On the other hand the map $E G \times G \rightarrow E G,(s, g) \mapsto g^{-1} s$, induces a homeomorphism $(E G \times G) / G \rightarrow E G$. It follows that $(E G \times G x) / G \cong E G$ and so $H_{G}^{q}(G x)$ is isomorphic to $H^{q}(E G)$ for every $q \in \mathbb{Z}$. Since $E G$ is contractible, we have $H^{q}(E G)=0$ for $q \in \mathbb{N}$. Thus

$$
H_{G}^{q}(G x)=0 \quad \text { for all } q \in \mathbb{N} .
$$

Let $W$ be an arbitrary neighborhood of $G x$. As $x \neq \Theta(x)$ there is a closed ball $B$ centered at $x$ such that $B \cap \Theta(B)=\emptyset$ and $N:=B \cup \Theta(B) \subset W$. Since $B$ and $\Theta(B)$ are disjoint, one can easily construct a $G$-equivariant strong deformation retraction $h:[0,1] \times N \rightarrow N$ of $N$ onto $G x$, namely, $h(t, y)=(1-t) x+t y$ if $y \in B$ and $h(t, y)=\Theta h\left(t, \Theta^{-1} y\right)$ otherwise. The map $h$ induces a strong deformation retraction $[0,1] \times(E G \times N) / G \rightarrow$ $(E G \times N) / G$ of $(E G \times N) / G$ onto $(E G \times G x) / G$. It follows that $H_{G}^{*}(G x)$ and $H_{G}^{*}(N)$ are isomorphic. Consequently, (3.3) implies that $H_{G}^{q}(N)=0$ for all $q \in \mathbb{N}$.

Definition 3.4. Let $\left(X, x_{0}\right)$ be a pointed $G$-space, i.e. assume that $\left(X,\left\{x_{0}\right\}\right)$ is a pair of $G$-spaces. Let $r \in \mathbb{N}_{0}$. We say that $\left(X, x_{0}\right)$ is of type 
$\Sigma_{G}^{r}$ if there is an $r$-dimensional inner product space $V$ on which $G$ acts orthogonally (i.e. $V$ is a $G$-orthogonal representation space), and $\left(X, x_{0}\right)$ is $G$ homotopy equivalent to the pointed $G$-space $\left(Z, z_{0}\right):=(D(V) / S(V),[S(V)])$, where $D(V)$ and $S(V)$ are the unit disk and the unit sphere of $V$, respectively, and the $G$-action on $\left(Z, z_{0}\right)$ is that induced by the $G$-action on $V$.

REMARK. Since every $G$-homotopy equivalence is an ordinary homotopy equivalence and since the pointed space $(D(V) / S(V),[S(V)])$ is homeomorphic (hence homotopy equivalent) to a pointed sphere $\left(S^{r}, s_{0}\right)$ it follows that every pointed $G$-space of type $\Sigma_{G}^{r}$ has the (ordinary) homotopy type $\Sigma^{r}$ of a pointed $r$-sphere. However, two pointed spaces of type $\Sigma_{G}^{r}$ need not be $G$-homotopy equivalent. To see this, let $V_{1}$ be the space $\mathbb{R}^{2}$ endowed with the antipodal $G$-action $(g, x) \mapsto(-1)^{g} x$, while $V_{2}$ be the space $\mathbb{R}^{2}$ endowed with the $G$-action $\left(g,\left(x_{1}, x_{2}\right)\right) \mapsto\left(x_{1},(-1)^{g} x_{2}\right)$. Then the pointed $G$-spaces

$$
\begin{aligned}
& \left(Z_{1}, z_{1}\right):=\left(D\left(V_{1}\right) / S\left(V_{1}\right),\left[S\left(V_{1}\right)\right]\right), \\
& \left(Z_{2}, z_{2}\right):=\left(D\left(V_{2}\right) / S\left(V_{2}\right),\left[S\left(V_{2}\right)\right]\right)
\end{aligned}
$$

are not $G$-homotopy equivalent. In fact, $G$-equivariant maps map $G$-fixed points to $G$-fixed points. Furthermore, the set of $G$-fixed points of $Z_{1}$ has two elements and hence is disconnected, while the set of $G$-fixed points of $Z_{2}$ is connected. It follows that whenever $f:\left(Z_{1}, z_{1}\right) \rightarrow\left(Z_{2}, z_{2}\right)$ and $g:\left(Z_{2}, z_{2}\right) \rightarrow$ $\left(Z_{1}, z_{1}\right)$ are $G$-equivariant and continuous then $h:=g \circ f:\left(Z_{1}, z_{1}\right) \rightarrow\left(Z_{1}, z_{1}\right)$ maps the two $G$-fixed points of $Z_{1}$ to the single $G$-fixed point of $Z_{1}$. Therefore, in particular, $h$ cannot be $G$-homotopic to the identity map on $\left(Z_{1}, z_{1}\right)$.

It follows from these considerations that, in contrast to the nonequivariant case, the symbol $\Sigma_{G}^{r}$ does not denote a single $G$-homotopy type, but, rather, a class of $G$-homotopy types.

Definition 3.5. Let $M$ be an arbitrary $H^{*}(B G)$-module. An element $u \in M$ is called nontorsion if $\omega^{m} * u \neq 0$ for all $m \in \mathbb{N}$. Here, $*$ denotes the multiplication $H^{*}(B G) \times M \rightarrow M$.

Proposition 3.6. Let $\left(N_{1}, N_{2}\right)$ be a pair of $G$-spaces such that the pair $\left(N_{1} / N_{2},\left[N_{2}\right]\right)$ is of type $\Sigma_{G}^{r}$ for some $r \in \mathbb{N}_{0}$. Let $q$ be the smallest nonnegative integer for which there is a nontorsion element $v \in H_{G}^{q}\left(N_{1}, N_{2}\right)$. Then $q=r$.

Proof. Let $V$ be as in Definition 3.4. It is clear (and also follows from Lemma 2 of [7]) that $H_{G}^{*}\left(N_{1}, N_{2}\right)$ and $H_{G}^{*}\left(N_{1} / N_{2},\left[N_{2}\right]\right)$ are isomorphic $H^{*}(B G)$-modules. Also, $H_{G}^{*}(D(V), S(V))$ and $H_{G}^{*}(D(V) / S(V),[S(V)])$ are isomorphic $H^{*}(B G)$-modules. Thus our assumption implies that

$$
H_{G}^{*}\left(N_{1}, N_{2}\right) \text { and } H_{G}^{*}(D(V), S(V)) \text { are isomorphic } H^{*}(B G) \text {-modules. }
$$

Since $R=\mathbb{Z}_{2}$ it follows that the vector bundle $\zeta:(E G \times V) / G \rightarrow B G$ induced by the projection $E G \times V \rightarrow E G$ is orientable over $R$. Thus Thom's 
isomorphism theorem (cf. Theorem 5.7.10 of [18]) implies that there is a $u \in H_{G}^{r}\left(D(V), S(V)\right.$ ) (the Thom class of $\zeta$ ) such that the map $H^{*}(B G) \rightarrow$ $H_{G}^{*+r}(D(V), S(V)), a \mapsto a * u$, is an $H^{*}(B G)$-module isomorphism. Together with (3.4) this implies that there is a $v \in H_{G}^{r}\left(N_{1}, N_{2}\right)$ such that the map $H^{*}(B G) \rightarrow H_{G}^{*+r}\left(N_{1}, N_{2}\right), a \mapsto a * v$, is an $H^{*}(B G)$-module isomorphism. Hence we conclude that $H_{G}^{p}\left(N_{1}, N_{2}\right)=0$ for all $p<r$. Thus the number $q$, if it exists, must satisfy $q \geq r$. To show that $q=r$ we just have to show that $v$ is nontorsion. If this is not true then $\omega^{m} * v=0$ for some $m \in \mathbb{N}$. This implies $\omega^{m}=0$. However, recalling that $H^{*}(B G)=\mathbb{Z}_{2}[\omega]$ and so, in particular, $\omega^{m} \neq 0$ for all $m \in \mathbb{N}$, we obtain a contradiction.

For the rest of this section assume that $E$ is a finite-dimensional inner product space and $G$ acts on $E$ orthogonally. Let $U \subset E$ be open and $G$-invariant.

Assume that $f \in C(U \rightarrow E)$ is $G$-equivariant and let $N$ be a $G$ invariant isolating neighborhood relative to $f$. Let $\varepsilon(f, N)$ be as in Proposition 3.6 of [12]. By the results of [7] and [12] it follows that whenever $f^{\prime}, f^{\prime \prime}: U \rightarrow E$ are arbitrary $G$-equivariant locally Lipschitzian maps with $\left|f^{\prime}-f\right|_{N}<\varepsilon(f, N)$ and $\left|f^{\prime \prime}-f\right|_{N}<\varepsilon(f, N)$, and $\left(N_{1}^{\prime}, N_{2}^{\prime}\right)\left(\operatorname{resp} .\left(N_{1}^{\prime \prime}, N_{2}^{\prime \prime}\right)\right)$ are arbitrary $G$-invariant index pairs in $N$ relative to the flow generated by $f^{\prime}$ (resp. $\left.f^{\prime \prime}\right)$, then the pointed spaces $\left(N_{1}^{\prime} / N_{2}^{\prime},\left[N_{2}^{\prime}\right]\right)$ and $\left(N_{1}^{\prime \prime} / N_{2}^{\prime \prime},\left[N_{2}^{\prime \prime}\right]\right)$ are $G$ homotopy equivalent. Moreover, the graded $H^{*}(B G)$-modules $H_{G}^{*}\left(N_{1}^{\prime}, N_{2}^{\prime}\right)$ and $H_{G}^{*}\left(N_{1}^{\prime \prime}, N_{2}^{\prime \prime}\right)$ are isomorphic. Thus we have a well defined $G$-homotopy type

$$
h_{G}(f, N):=h_{G}\left(N_{1}^{\prime} / N_{2}^{\prime},\left[N_{2}^{\prime}\right]\right),
$$

which we call the $G$-equivariant Conley index of $(f, N)$. We also have a well defined $H^{*}(B G)$-module isomorphism type

$$
H_{G}^{*}(f, N):=H_{G}^{*}\left(N_{1}^{\prime}, N_{2}^{\prime}\right),
$$

which we call the $G$-equivariant cohomological Conley index of $(f, N)$. Actually $h_{G}(f, N)$ (resp. $\left.H_{G}^{*}(f, N)\right)$ depends only on the invariant set $S=$ $\operatorname{Inv}(f, N)$ and not on the choice of an isolating neighborhood. Thus we have a well defined $G$-equivariant Conley index $h_{G}(f, S):=h_{G}(f, N)$ (resp. the $G$-equivariant cohomological Conley index $\left.H_{G}^{*}(f, S):=H_{G}^{*}(f, N)\right)$ where $S$ is a compact, $G$-invariant, and isolated invariant set relative to $f$, and $N$ is an arbitrary $G$-invariant isolating neighborhood of $S$. The fact that we use the same symbols to denote the index of an isolating neighborhood and the index of an isolated invariant set should not lead to confusion.

We say that $h_{G}(f, S)$ (or $h_{G}(f, N)$ ) is of type $\Sigma_{G}^{r}$ if (in the above notation) $\left(N_{1}^{\prime} / N_{2}^{\prime},\left[N_{2}^{\prime}\right]\right)$ is of type $\Sigma_{G}^{r}$. Again this property is independent of the choice of $f^{\prime}$ and $\left(N_{1}^{\prime}, N_{2}^{\prime}\right)$.

We have the following elementary 
Proposition 3.7. Let $f: E \rightarrow E$ be linear, $G$-equivariant and symmetric with respect to the inner product on $E$. Assume also that $f$ has $r$ positive and $\operatorname{dim} E-r$ negative eigenvalues, counted with multiplicities. Then $\{0\}$ is a $G$-invariant isolated invariant set relative to $f$ and $h_{G}(f,\{0\})$ is of type $\Sigma_{G}^{r}$.

We can now state and prove the following abstract multiplicity result. This result is based on Theorem 5.1 of [11], which, in turn, is based on ideas from $[8]$.

Theorem 3.8. Let $U \subset E$ be open, $G$-invariant and $f \in C(U \rightarrow E)$ be $G$-equivariant. Suppose that $N \subset U$ is a $G$-invariant isolating neighborhood relative to $f$. Let $k \in \mathbb{N}$, for every $r \in \llbracket 1, k \rrbracket$ let $W_{r} \subset N$ be a $G$-invariant isolating neighborhood relative to $f$, set $M_{r}:=\operatorname{Inv}\left(f, W_{r}\right)$ and suppose that $\left(M_{r}\right)_{r=1}^{k}$ is a Morse decomposition of $S:=\operatorname{Inv}(f, N)$ relative to $f$ in the sense of Definition 2.4. Suppose that there is a $\nu \in \llbracket 1, k \rrbracket$ and numbers $p_{0}, p_{\infty} \in \mathbb{N}_{0}$ such that $h_{G}\left(f, W_{\nu}\right)$ is of type $\Sigma_{G}^{p_{0}}$ and $h_{G}(f, N)$ is of type $\Sigma_{G}^{p_{\infty}}$. Moreover, suppose that $\omega * H_{G}^{*}\left(W_{r}\right)=0$ for all $r \in \llbracket 1, k \rrbracket$ with $r \neq \nu$. Then

$$
k-1 \geq\left|p_{0}-p_{\infty}\right|
$$

Remark. Despite some (deceptive) similarity in the notations we should notice a fundamental difference between this theorem and Theorem 5.1 of [11]: here we consider only a finite-dimensional ODE and, in particular, we do not use the concept of a $G$-spectrum, introduced in [11].

Proof. Recall that the space $E$ is finite-dimensional. By Theorem 4.6 of [4] there is an $\varepsilon>0, \varepsilon \leq \varepsilon(f, N)$, such that whenever $f^{\prime} \in C(U \rightarrow E)$ satisfies $\left|f-f^{\prime}\right|_{N}<\varepsilon$ then $N$ and $W_{r}, r \in \llbracket 1, k \rrbracket$, are isolating neighborhoods relative to $f^{\prime}$ and $\left(\operatorname{Inv}\left(f^{\prime}, W_{r}\right)\right)_{r=1}^{k}$ is a Morse decomposition of $\operatorname{Inv}\left(f^{\prime}, N\right)$ relative to $f^{\prime}$. Clearly, $h_{G}\left(f^{\prime}, W_{\nu}\right)$ is of type $\Sigma_{G}^{p_{0}}$ and $h_{G}\left(f^{\prime}, N\right)$ is of type $\Sigma_{G}^{p_{\infty}}$. Choosing, if necessary, $f^{\prime}$ to be $G$-equivariant and locally Lipschitzian we may thus assume, with no loss of generality, that $f$ itself is locally Lipschitzian. Therefore we can consider the flow $\pi$ defined by the ODE $\dot{x}=f(x)$.

Let $\left(A_{r}\right)_{r=0}^{k}$ be the attractor filtration with respect to $\pi$ associated with the Morse decomposition $\left(M_{r}\right)_{r=1}^{k}$. For every $r \in \llbracket 1, k \rrbracket$ the pair $\left(A_{r-1}, M_{r}\right)$ is a $G$-invariant attractor-repeller pair in $A_{r}$. Therefore there is a $G$-invariant index triple $\left(N_{1}^{r}, N_{2}^{r}, N_{3}^{r}\right)$ for the pair $\left(A_{r-1}, M_{r}\right)$, relative to $A_{r}$, with $N_{1}^{r} \supset N_{2}^{r} \supset N_{3}^{r}$. (Cf. [5], [16] and in particular Definition III 2.2 of [15].) Choose any $G$-invariant open set $V$ in $E$ with $A_{r-1} \subset V \subset N_{2}^{r}$. Then $\left(N_{1}^{r} \backslash V, N_{2}^{r} \backslash V\right)$ is a $G$-invariant index pair in $N_{1}^{r} \backslash V$. By excision, the $H^{*}(B G)$-module $H_{G}^{*}\left(N_{1}^{r} \backslash V, N_{2}^{r} \backslash V\right)$, representing $H_{G}^{*}\left(f, M_{r}\right)$, is isomorphic to the $H^{*}(B G)$-module $H_{G}^{*}\left(N_{1}^{r}, N_{2}^{r}\right)$. Using the long exact $G$-equivariant 
cohomology sequence of the triple $\left(N_{1}^{r}, N_{2}^{r}, N_{3}^{r}\right)$ of Proposition 3.1 we thus obtain a well defined long exact sequence

$$
\ldots \stackrel{\delta^{*}}{\rightarrow} H_{G}^{*}\left(f, M_{r}\right) \stackrel{j^{*}}{\rightarrow} H_{G}^{*}\left(f, A_{r}\right) \stackrel{i^{*}}{\rightarrow} H_{G}^{*}\left(f, A_{r-1}\right) \stackrel{\delta^{*}}{\rightarrow} H_{G}^{*}\left(f, M_{r}\right) \stackrel{j^{*}}{\rightarrow} \ldots
$$

of $R$-module homomorphisms. Proposition 3.1 and the fact that $G=\mathbb{Z}_{2}$ (and so $-g=g$ for $g \in G)$ imply that the maps $j^{*}, i^{*}$ and $\delta^{*}$ are $H^{*}(B G)$-module homomorphisms.

Let $r \in \llbracket 1, k \rrbracket$ and $r \neq \nu$. Then there is a $G$-invariant index pair $\left(N_{1}^{\prime}, N_{2}^{\prime}\right)$ in $W_{r}$ relative to the flow $\pi$. It follows from our assumptions and Proposition 3.2 that $\omega * H_{G}^{*}\left(N_{1}^{\prime}, N_{2}^{\prime}\right)=0$ and so $\omega * H_{G}^{*}\left(f, M_{r}\right)=0$.

We first claim that

$$
\omega^{r} * H_{G}^{*}\left(f, A_{r}\right)=0, \quad r \in \llbracket 0, \nu-1 \rrbracket .
$$

In fact, $A_{0}=\emptyset$ so $H_{G}^{*}\left(f, A_{r}\right)=0$ and thus $\omega^{r} * H_{G}^{*}\left(f, A_{r}\right)=0$ for $r=0$. Let $r \in \llbracket 1, \nu-1 \rrbracket$ and suppose that $\omega^{r-1} * H_{G}^{*}\left(f, A_{r-1}\right)=0$. Whenever $u \in H_{G}^{*}\left(f, A_{r}\right)$ then $i^{*}(u) \in H_{G}^{*}\left(f, A_{r-1}\right)$, so $0=\omega^{r-1} * i^{*}(u)=i^{*}\left(\omega^{r-1} * u\right)$, and so the exactness of the sequence (3.5) implies that $j^{*}(z)=\omega^{r-1} * u$ for some $z \in H_{G}^{*}\left(f, M_{r}\right)$. Since $r \neq \nu$ we obtain $\omega * z=0$ and so $\omega^{r} * u=$ $\omega * j^{*}(z)=j^{*}(\omega * z)=0$. This proves (3.6) by induction.

For $r \in \llbracket 0, k \rrbracket$ let $n_{r}$ be the smallest nonnegative number $n$ such that $H_{G}^{n}\left(f, A_{r}\right)$ contains a nontorsion element. We claim that for all $r \in \llbracket \nu, k \rrbracket$ the number $n_{r}$ is defined and

$$
\begin{gathered}
p_{0}-(\nu-1) \leq n_{\nu} \leq p_{0}, \\
n_{r-1} \leq n_{r} \leq n_{r-1}+1, \quad r \in \llbracket \nu+1, k \rrbracket .
\end{gathered}
$$

By Proposition 3.6 there is a nontorsion element $u \in H_{G}^{p_{0}}\left(f, M_{\nu}\right)$. We claim that $j^{*}(u)$ is nontorsion. Indeed, if $0=\omega^{m} * j^{*}(u)=j^{*}\left(\omega^{m} * u\right)$ then by exactness in (3.5) there is an $x \in H_{G}^{*}\left(f, A_{\nu-1}\right)$ with $\omega^{m} * u=\delta^{*}(x)$. Now (3.6) implies that $\omega^{\nu-1} * x=0$ and so we obtain $\omega^{\nu-1+m} * u=\omega^{\nu-1} * \delta(x)=$ $\delta\left(\omega^{\nu-1} * x\right)=0$, a contradiction. Since $j^{*}(u) \in H_{G}^{p_{0}}\left(f, A_{\nu}\right)$ it follows that $n_{\nu}$ is defined and, indeed, $n_{\nu} \leq p_{0}$. Now let $z \in H_{G}^{n_{\nu}}\left(f, A_{\nu}\right)$ be nontorsion. Formula (3.6) implies that $i^{*}\left(\omega^{\nu-1} * z\right)=0$, so $\omega^{\nu-1} * z=j^{*}(x)$ for some $x \in H_{G}^{*}\left(f, M_{\nu}\right)$ with

$$
\operatorname{dim} x=\operatorname{dim}\left(\omega^{\nu-1} * z\right)=(\nu-1)+n_{\nu} .
$$

Since $\omega^{m} * x=0$ implies $\omega^{\nu-1+m} * z=0$, we see that $x$ is nontorsion and so, by Proposition 3.6, $\operatorname{dim} x \geq p_{0}$. Thus (3.9) implies that $p_{0} \leq(\nu-1)+n_{\nu}$ and (3.7) is proved. Now suppose $r \in \llbracket \nu+1, k \rrbracket$ is such that $n_{r-1}$ is defined. Let $z \in H_{G}^{n_{r-1}}\left(f, A_{r-1}\right)$ be nontorsion. Since $r \neq \nu$, we have $\delta(\omega * z)=$ $\omega * \delta^{*}(z)=0$, so there is an $x \in H_{G}^{*}\left(f, A_{r}\right)$ with $i^{*}(x)=\omega * z$ and

$$
\operatorname{dim} x=\operatorname{dim}(\omega * z)=1+n_{r-1} .
$$


As $x$ is clearly nontorsion, it follows from (3.10) that $n_{r}$ is defined and $n_{r} \leq n_{r-1}+1$. Now let $x \in H_{G}^{n_{r}}\left(f, A_{r}\right)$ be nontorsion. We claim that $i^{*}(x)$ is nontorsion. Indeed, $\omega^{m} * i^{*}(x)=0$ implies that $\omega^{m} * x=j^{*}(z)$ for some $z \in H_{G}^{*}\left(f, M_{r}\right)$. It follows that $\omega * z=0$, so $\omega^{m+1} * x=0$, a contradiction. Thus $n_{r-1} \leq \operatorname{dim} i^{*}(x)=\operatorname{dim} x=n_{r}$. Altogether we see that (3.8) holds.

Iterating (3.8), using (3.7) and noting that, by Proposition 3.6, $p_{\infty}=n_{k}$ we obtain $p_{\infty}=n_{k} \leq n_{\nu}+(k-\nu) \leq p_{0}+(k-1)$ so

$$
p_{\infty}-p_{0} \leq k-1 \text {. }
$$

Using (3.8) and (3.7) again we also obtain $p_{\infty}=n_{k} \geq n_{\nu} \geq p_{0}-(\nu-1) \geq$ $p_{0}-(k-1)$, so

$$
p_{\infty}-p_{0} \geq-(k-1) .
$$

Now formulas (3.11) and (3.12) imply that $(k-1) \geq\left|p_{0}-p_{\infty}\right|$.

4. A proof of the Angenent-van der Vorst conjecture. In this section we will prove Conjecture A stated in the introduction. Throughout this section we use the notation of [13]. We will use the abstract multiplicity result established in the preceding section as well as some basic results from [13].

Assume Hypothesis 1.1(1)-(3) as well as the following hypotheses:

Hypothesis 4.1. $H(-\xi,-\eta, x)=H(\xi, \eta, x)$ for all $(\xi, \eta, x) \in \mathbb{R} \times \mathbb{R} \times \bar{\Omega}$.

HYPOTHESIS 4.2. $z_{0}=0$ is a hyperbolic solution of (1.1) with renormalized Morse index $\gamma \in \mathbb{Z}$.

For the rest of this section we consider the space $E$ as a $G$-space with respect to the antipodal $G$-action $(g, z) \mapsto(-1)^{g} z, g \in G=\mathbb{Z}_{2}, z \in E$. We then have the following $G$-equivariant Linearization Principle.

Theorem 4.3. Assume Hypotheses 1.1(1)-(3), 4.1 and 4.2. Then the $G$ equivariant Conley index $h_{G}\left(L+K_{1},\{0\}\right)_{l}$ is of type $\Sigma_{G}^{\gamma+l}$ for all l sufficiently large.

Proof. Let $K_{\operatorname{lin}}=K_{\text {lin }, 0}$. It is shown in the proof of Theorem 2.9 of [13] that there is a closed ball $N$ at zero such that for every $\theta \in[0,1]$ the set $N$ is an isolating neighborhood of $\{0\}$ relative to $L+\theta K_{1}+(1-\theta) K_{\operatorname{lin}}$. Since $N$ is $G$-invariant and the homotopy $[0,1] \times E \rightarrow E,(\theta, z) \mapsto L z+$ $\left(\theta K_{1}+(1-\theta) K_{\text {lin }}\right)(z)$, is $G$-equivariant, the $G$-homotopy invariance of the $G$-equivariant $\mathcal{L S}$-Conley index (see [12]) implies that

$$
\begin{aligned}
h_{G}\left(L+K_{1}, N\right)_{l} & =h_{G}\left(L+K_{\operatorname{lin}}, N\right)_{l} \\
& =h_{G}\left(L^{l}+\left(P^{l} \circ K_{\operatorname{lin}}\right)_{\mid E^{l}}, N \cap E^{l}\right) \\
& =h_{G}\left(L^{l}+\left(P^{l} \circ K_{\operatorname{lin}}\right)_{\mid E^{l}},\{0\}\right)
\end{aligned}
$$


for all $l$ large enough. Since the map $L+K_{\operatorname{lin}}$ is injective, it follows that for all $l$ large enough the linear map $L^{l}+\left(P^{l} \circ K_{\text {lin }}\right)_{\mid E^{l}}$ is injective, and by Theorems 2.2 and 2.3 this map has $\gamma+l$ positive eigenvalues (counted with multiplicities). Thus, by Proposition $3.7, h_{G}\left(L^{l}+\left(P^{l} \circ K_{\operatorname{lin}}\right)_{\mid E^{l}},\{0\}\right)$ is of type $\Sigma_{G}^{\gamma+l}$ for all $l$ large enough. This together with (4.1) completes the proof of the theorem.

Theorem 4.4. Assume Hypotheses 1.1, 4.1 and 4.2. Define $S$ to be the set of all points $z_{0} \in E$ for which there is a bounded solution $z: \mathbb{R} \rightarrow E$ of (1.2) such that $z(0)=z_{0}$. Then $S$ is a $G$-invariant isolated invariant set relative to $L+K_{1}$ and $h_{G}\left(L+K_{1}, S\right)_{l}$ is of type $\Sigma_{G}^{l}$ for all l sufficiently large.

Proof. The set $S$ is obviously $G$-invariant. Let $N$ be the closed ball at zero with radius $2 M$ where $M$ is as in Proposition 2.12 of [13]. Then that proposition implies that for every $\theta \in[0,1]$ the set $N$ is an isolating neighborhood relative to $L+\theta K_{1}$. Since $N$ is $G$-invariant and the homotopy $[0,1] \times E \rightarrow E,(\theta, z) \mapsto L z+\theta K_{1}(z)$, is $G$-equivariant, the $G$-homotopy invariance of the $G$-equivariant $\mathcal{L S}$-Conley index implies that

$$
\begin{aligned}
h_{G}\left(L+K_{1}, N\right)_{l} & =h_{G}(L, N)_{l}=h_{G}\left(L^{l}, N \cap E^{l}\right) \\
& =h_{G}\left(L^{l},\{0\}\right) \quad \text { for all } l \text { large enough. }
\end{aligned}
$$

For all $l$ the linear map $L^{l}$ is injective and has $l$ positive eigenvalues (counted with multiplicities). Thus, by Proposition $3.7, h_{G}\left(L^{l},\{0\}\right)$ is of type $\Sigma_{G}^{l}$ for all $l \in \mathbb{N}$. This together with (4.2) completes the proof of the theorem.

We can now prove the Angenent-van der Vorst conjecture:

Theorem 4.5. Assume Hypotheses 1.1, 4.1 and 4.2. Then system (1.1) has at least $2|\gamma|$ nontrivial solutions.

Proof. If (1.1) has infinitely many solutions then we are done. Therefore, suppose that the set $\mathcal{E}$ of solutions of (1.1) is finite. Let $S$ be as in Theorem 4.4 and $N$ be a $G$-invariant isolating neighborhood of $S$ relative to $f:=L+K_{1}$. Let $\mathcal{P}$ be the set of all $G$-orbits of elements of $\mathcal{E}$ and $k \in \mathbb{N}$ be the cardinality of $\mathcal{P}$. By Hypothesis 4.1, the Lyapunov function $\Phi_{1}$ of the equation $\dot{z}=f(z)$ is constant on $G$-orbits. Thus we can order $\mathcal{P}$ in such a way that $\mathcal{P}=\left\{M_{r} \mid r \in \llbracket 1, k \rrbracket\right\}$ where $\left(M_{r}\right)_{r=1}^{k}$ is a Morse decomposition of $S=\operatorname{Inv}(f, N)$ relative to $f$, in the sense of Definition 2.4. There is a $\nu \in \llbracket 1, k \rrbracket$ such that $M_{\nu}=\{0\}$ while for all $r \neq \nu$ the set $M_{r}$ has two elements. It follows from Proposition 3.3 that whenever $r \neq \nu$, then $M_{r}=\operatorname{Inv}\left(f, W_{r}\right)$ for some $G$-invariant neighborhood $W_{r} \subset N$ of $M_{r}$ with $\omega * H_{G}^{*}\left(W_{r}\right)=0$. Choose $W_{\nu} \subset N$ to be an arbitrary $G$-invariant neighborhood of $M_{\nu}$ with $\operatorname{Inv}\left(f, W_{\nu}\right)=M_{\nu}$. For $l \in \mathbb{N}$ define the map $f^{l}: U \cap E^{l} \rightarrow E^{l}$ by $z \mapsto L^{l} z+P^{l} K_{1}(z)$. An application of Theorem 4.15 
of [4] shows that there is an $l_{1} \in \mathbb{N}$ such that whenever $l \geq l_{1}$ then the sets $N \cap E^{l}$ and $W_{r} \cap E^{l}, r \in \llbracket 1, k \rrbracket$, are isolating neighborhoods relative to $f^{l}$ and $\left(\operatorname{Inv}\left(f^{l}, W_{r} \cap E^{l}\right)\right)_{r=1}^{k}$ is a Morse decomposition of $\operatorname{Inv}\left(f^{l}, N \cap E^{l}\right)$ relative to $f^{l}$. Using Theorems 4.3 and 4.4 we further find an $l_{2} \in \mathbb{N}$ such that whenever $l \geq l_{2}$ then $h_{G}\left(f^{l}, W_{\nu} \cap E^{l}\right)$ is of type $\Sigma_{G}^{\gamma+l}$ and $h_{G}\left(f^{l}, N \cap E^{l}\right)$ is of type $\Sigma_{G}^{l}$.

Fix $l \geq \max \left(l_{1}, l_{2}\right)$. Set $p_{0}:=\gamma+l, p_{\infty}:=l, U^{\prime}=E^{\prime}:=E^{l}, f^{\prime}:=$ $f^{l}, N^{\prime}:=N \cap E^{l}, S^{\prime}:=\operatorname{Inv}\left(f^{l}, N \cap E^{l}\right)$, and moreover $W_{r}^{\prime}:=W_{r} \cap E^{l}$, $M_{r}^{\prime}:=\operatorname{Inv}\left(f^{l}, W_{r} \cap E^{l}\right)$ for $r \in \llbracket 1, k \rrbracket$. Using Proposition 3.2 we see that $\omega * H_{G}^{*}\left(W_{r}^{\prime}\right)=0$ for every $r \neq \nu$.

Now an application of Theorem 3.8 implies that $k-1 \geq\left|p_{0}-p_{\infty}\right|=|\gamma|$. This means that there are at least $2|\gamma|$ nontrivial solutions of system (1.1).

\section{References}

[1] S. Angenent and R. van der Vorst, A superquadratic indefinite elliptic system and its Morse-Conley-Floer homology, Math. Z. 231 (1999), 203-248.

[2] T. Bartsch, Topological Methods for Variational Problems with Symmetry, Lecture Notes in Math. 1560, Springer, Berlin, 1993.

[3] G. E. Bredon, Introduction to Compact Transformation Groups, Academic Press, New York, 1972.

[4] M. C. Carbinatto and K. P. Rybakowski, Morse-decompositions in the absence of uniqueness, Topol. Methods Nonlinear Anal. 18 (2001), 305-342.

[5] C. C. Conley, Isolated Invariant Sets and the Morse Index, CBMS Regional Conf. Ser in Math. 38, Amer. Math. Soc., Providence, 1978.

[6] T. tom Dieck, Transformation Groups, de Gruyter, Berlin, New York, 1987.

[7] A. Floer, A refinement of the Conley index and its application to the stability of hyperbolic invariant sets, Ergodic Theory Dynam. Systems 7 (1987), 93-103.

[8] A. Floer and E. Zehnder, The equivariant Conley index and bifurcations of periodic solutions of Hamiltonian systems, ibid. 8* (1988), 87-97.

[9] K. Gęba, M. Izydorek and A. Pruszko, The Conley index in Hilbert spaces, Studia Math. 134 (1999), 217-233.

[10] M. Izydorek, A cohomological Conley index in Hilbert spaces and applications to strongly indefinite problems, J. Differential Equations 170 (2001), 22-50.

[11] - Equivariant Conley index in Hilbert spaces and applications to strongly indefinite problems, Nonlinear Anal. 51 (2002), 33-66.

[12] M. Izydorek and K. P. Rybakowski, On the Conley index in Hilbert spaces in the absence of uniqueness, Fund. Math. 171 (2002), 31-52.

[13] - - - The Conley index in Hilbert spaces and a problem of Angenent and van der Vorst, ibid. 173 (2002), 77-100.

[14] J. Robbin and D. Salamon, The spectral flow and the Morse index, Bull. London Math. Soc. 27 (1995), 113-140.

[15] K. P. Rybakowski, The Homotopy Index and Partial Differential Equations, Springer, Berlin, 1987.

[16] K. P. Rybakowski and E. Zehnder, On a Morse equation in Conley's index theory for semiflows on metric spaces, Ergodic Theory Dynam. Systems 5 (1985), 123-143. 
[17] M. Schwarz, Morse Homology, Birkhäuser, 1993.

[18] E. Spanier, Algebraic Topology, McGraw-Hill, New York, 1966.

Department of Technical Physics and Applied Mathematics

Gdańsk University of Technology

Narutowicza 11/12

80-952 Gdańsk, Poland

E-mail: izydorek@mifgate.pg-gda.pl

Fachbereich Mathematik

Universität Rostock

Universitätsplatz 1

18055 Rostock, Germany

E-mail: krzysztof.rybakowski@mathematik.uni-rostock.de

Received 27 May 2002;

in revised form 20 January 2003 